Erwerbsarbeit im Wandel: Impulse für eine nachhaltige Lebensführung?

\section{Flexibilisierung als Barriere}

\author{
In den Debatten um die Wege zu einer zukunftsfähigen Entwicklung haben \\ individuelle Konsummuster einen zentralen Stellenwert. Ein strategischer \\ Ansatz- punkt liegt folglich darin, Leitbilder für ressourcensparende Konsum- \\ formen vor- zuschlagen. Doch wie verträglich sind solche Leitbilder mit den \\ heutigen Arbeits- und Lebensbedingungen breiter Schichten der Bevölkerung? \\ Anders als vielfach vermutet erleichtert der Wandel der Erwerbsarbeit \\ eine nachhaltige Lebensföhrung nur bedingt.
}

$\mathrm{L}$

Von Volker Hielscher eitbilder können nur dann eine Relevanz

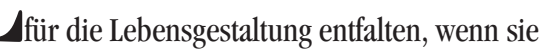
sowohl an die Vorstellungswelten als auch an die Rahmenbedingungen und strukturellen Zwänge der individuellen Lebensführung anknüpfen. Fragwïrdig ist zum Beispiel, ob der Vorschlag, die Wohlstandsvorstellung entlang des Prinzips „gut leben statt viel haben" auszurichten, an die Kontexte breiter Bevölkerungsschichten anschließen kann. Darüber hinaus erfassen diese Leitbilder nur sehr bedingt die Dynamik des gesellschaftlichen Wandels, vor allem im Hinblick auf die Veränderungen der Erwerbsarbeit und auf fortdauernde Massenarbeitslosigkeit sowie den Abbau sozialer Sicherungen. In einer historischen Situation zunehmender Unsicherheiten und einer prekärer werdenden Existenzsicherung laufen die Leitbilder für eine ökologische Lebensführung Gefahr, als normativer Appell lediglich in bestimmten Vorreitergruppen Gehör zu finden, ohne aber eine nennenswerte Bedeutung im Alltag breiterer Bevölkerungsschichten zu erhalten. An der Schnittstelle zwischen neuen Entwicklungsdynamiken der Erwerbsarbeit und den Anforderungen der Nachhaltigkeit hat ein Forschungsprojekt des Wissenschaftszentrums Berlin für Sozialforschung angesetzt (1). Analysiert wurden die Folgewirkungen verkürzter und flexibilisierter Arbeitszeiten auf die Lebensführung und Lebensqualität der Beschäftigten. Der Ansatz der Lebensführung (2) greift über die Analyse einzelner Handlungsbereiche (wie beispielsweise Einkaufs- und Konsumverhalten) insofern hinaus, als dass die Grundmuster und Arrangements des praktischen Alltagslebens betrachtet werden, welche die Menschen aktiv herstellen müssen. Diese sind in Arbeit und Lebenswelt stark durch Systemvorgaben und Gelegenheitsstrukturen geprägt. Ein besonderes Augenmerk ökologischen und sozialen Folgewirkungen waren von den Tarifparteien zunächst allerdings nicht mitbedacht worden.

\section{Arbeitszeitpolitik und nachhaltige Lebensführung}

Die bisher nicht gestalteten Nebenfolgen und die nicht reflektierten Wechselwirkungen zwischen neuen Arbeitszeitmustern und nachhaltiger Lebensführung waren der Ausgangspunkt für die folgenden Hypothesen:

- Die umfassende Arbeitszeitverkürzung bietet einen Zuwachs an persönlich verfügbarer Zeit, die eine Ressource darstellen kann: für breitere sozial-ökologische Information und Bildung, für die Reflexion der eigenen Lebensführung und um Änderungen des Alltagsverhaltens auszuprobieren.

- Die Einschränkung des verfügbaren Einkommens erzeugt einen Druck, den kommerziellen Konsum einzuschränken und die eigenen Konsumgewohnheiten zu überdenken.

- Ein Teil der neuen Arbeitsformen erhebt den Anspruch, mehr Raum für die individuelle Identifikation und für die Übernahme von Verantwortung für die Arbeitsprozesse zu eröffnen. Dies könnte auch das generelle Verantwortungsbewusstsein für soziale und ökologische Folgen des eigenen Verhaltens stärken.

- In Zeiten der Beschäftigungskrise erhöht der Tarifvertrag die Arbeitsplatzsicherheit. Diese Sicherheit bietet eine Grundlage für eine höhere Motivation zu reflektiertem und innovativem Verhalten in Arbeit und Privatleben.

Der Tarifvertrag hatte in seiner ursprünglichen Regelung Rahmenbedingungen geschaffen, die in der Nachhaltigkeitsdebatte für die Entwicklung von Zeitwohlstand (statt Güterreichtum) vorausgesetzt werden. Mit einer Befragung der Beschäftigten und mittels Experteninterviews haben wir geprüft, vor welche Anforderungen die Lebensführung der Beschäftigten neu gestellt wird und inwieweit soziale und ökologische Überlegungen bei einer Umstellung der Lebensführung eine Rolle spielen.

\section{Umstellungspotenzial ungenutz $\dagger$}

Die Resultate zeigen, dass die möglichen positiven Effekte der Arbeitszeitverkürzung ein Potenzial sind, das bisher nur wenig genutzt wurde, während die Negativeffekte der Flexibilisierung vergleichsweise stark durchgeschlagen sind.

Die Arbeitszeitverkürzung wurde von vielen Beschäftigten als eine Entlastung und Entspannung 
des Alltags gesehen. Sie war willkommen, um die Dinge etwas geruhsamer anzugehen und mehr Zeit für die Familie zu haben. Die zusätzliche freie Zeit versickerte quasi; sie wurde kaum als ein zu gestaltendes neues ,Zeitpaket“ gesehen. Ebenso wenig wurden Ansprüche an zusätzlicher Zeit für eigensinnige, bisher zurückgestellte Bedürfnisse artikuliert. Eine Reflexion darüber, wie nun das Leben mit mehr Zeit (und weniger Einkommen) gestaltet werden könnte, ist von selbst kaum in Gang gesetzt worden.

Insofern hat die Arbeitszeitverkürzung als solche die vorhandenen Arrangements der Lebensfiuhrung erst einmal stabilisiert. Hobbies und Freizeitaktivitäten wurden gelegentlich ausgebaut, allerdings nicht grundlegend verändert. Analog dazu wurde ein ökologisch motiviertes Engagement und Alltagsverhalten nur bei wenigen Personen befördert, für die Ökologie und Umweltschutz bereits vor der Arbeitszeitveränderung einen wichtigen individuellen Stellenwert hatten.

\section{- Neve Barriere Flexibilisierung?}

Die Flexibilisierung der Arbeit, also die Auflösung und Beseitigung von arbeitsweltlichen Strukturen, Regelungen, Gewohnheiten und Orientierungen ist seit den neunziger Jahren der zentrale Hebel für die Modernisierung der Erwerbsarbeit.

Eine derartige Flexibilisierung stellt eine Reihe neuer Anforderungen an die Lebensfiuhrung der Beschäftigten, zudem beeinflusst sie unmittelbar die Chancen für solche Umstellungen von Konsumgewohnheiten, die aus ökologischer Perspektive geboten scheinen:

1. Aus der Vervielfachung der Arbeitszeitmodelle und aus der variablen täglichen und wöchentlichen Arbeitszeit für viele Beschäftigtengruppen resultierten massive Koordinationsprobleme innerhalb des Betriebs und im privaten Leben der Beschäftigten. Aufgrund der individuell jeweils unterschiedlichen Arbeitszeiten zerfielen viele Fahrgemeinschaften; der ÖPNV erlitt schwere Einbrïche, weil das Angebot nicht mehr auf definierbare „Rush-hours“ zum Schichtwechsel abgestellt werden konnte. Die private Koordination im Freundes- und Bekanntenkreis ist erschwert worden, weil die etablierten Rhythmen und Sozialzeiten durch die unterschiedlichen Zeitmuster zerschnitten worden sind. Aus dem gleichen Grund wurde soziales und ökologisches Engagement in Vereinen, Verbänden und Initiativen erschwert.

\section{Permanenter Abwägungsprozess}

2. Durch die Flexibilisierung und die notwendige individuelle Mitgestaltung der Arbeitszeit werden die Beschäftigten gezwungen, ein individuelles Zeitmanagement $\mathrm{zu}$ entwickeln. Sie müssen einen komplexen und permanenten Abwägungsprozess zwischen den betrieblichen Anforderungen und den persönlichen Bedürfnissen und Interessen leisten. Insofern hat die Flexibilisierung ambivalente Folgen für die Lebensführung: Während einerseits prinzipiell Spielräume für eine verbesserte Abstimmung zwischen privatem Leben und Beruf geschaffen werden, wächst andererseits die Komplexität der Anforderungen und Interessen, die immer wieder neu ausbalanciert werden müssen.

\section{- Kernprobleme für Öko-Konsum}

Unter diesen Rahmenbedingungen sind ökologisch motivierte Ansprïche an das Konsumverhalten eine zusätzliche Anforderungsstruktur, die an die Lebensführung der Individuen herangetragen wird. Aus der Perspektive eines nachhaltigen Konsums ergeben sich mindestens zwei zentrale Probleme. Zum einen können diese Anforderungen trotz eines weit verbreiteten Bewusstseins um die Bedeutung der ökologischen Gefährdung ausschließlich als eine weitere Belastung in einer ohnehin schon gesteigerten Situation von Alltagsstress wahrgenommen werden - Reaktionen wie Nichtbeachtung oder offene Abwehr sind also nicht auszuschließen. Zweitens stehen viele Vorschläge zum nachhaltigen Konsum vor dem Paradox, dass in der flexibilisierten und individualisierten Gesellschaft der Bedarf an permanenter Verfügbarkeit wächst und dadurch gerade diejenigen sozialen Rhythmen labiler werden, auf denen Ansätze von stärker kollektiven Nutzungformen basieren. So entzieht sich vielen Beschäftigten mit flexiblen und atypischen Arbeitszeiten die Möglichkeit, den ÖPNV mit seinen starren Angebotsschemata oder das Automobil in einer Fahrgemeinschaft kollektiv zu nutzen. Ebenso wächst der Bedarf an die Verfügbarkeit von Rund-um-die-Uhr-Dienstleistungen, technischer Haushaltsausstattung (Beispiel: Mikrowelle zum Essen-Aufwärmen) und von Energie.

\section{- Hemmnis Ungewissheit}

3. Die Flexibilisierung hat nicht nur Folgewirkungen im Alltag, auch in der langfristigen Perspektive wächst die Ungewissheit, welche individuelle Arbeitszeit Bestand haben wird und welches Ein- kommen erzielt werden kann. Im Zusammenspiel mit der langfristigen Auflösung des Normalarbeitsverhältnisses und mit der allgemeinen Arbeitsmarktsituation wächst die Zukunftsunsicherheit. Für eine (sozial-ökologisch) motivierte Veränderung der Lebensfuihrung und für eine neue Gewichtung von Erwerbsarbeit, freier Zeit und materiellem Wohlstand existiert also keine gesicherte Perspektive. Statt eine Reflexion der eigenen Lebensfuihrung zu befördern, mündet die Ungewissheit angesichts der sozialen Krise für viele Beschäftigte in Ängste vor sozialem Abstieg. Trotz der Beschäftigungsgarantie wurde die soziale Zukunftsentwicklung weitaus skeptischer eingeschätzt als die ökologische Entwicklung. Der Druck zur Einschränkung durch die Einkommensabsenkung wurde als krisenbedingter und aufgezwungener Verzicht gesehen - hier waren kaum Ansatzpunkte zu entdecken für eine positive Interpretation im Sinne von Suffizienzstrategien, die Leitbilder von ,weniger haben und gut leben“ propagieren. In der Lebensperspektive dominierte das Beharren auf einmal gesetzten Lebensvorstellungen und die Bestrebung, das erreichte Wohlstandsniveau zu sichern.

\section{- Ökologisches Handeln im Alltag}

Die Entwicklungen im Arbeitsbereich und deren Folgewirkungen haben in der Praxis die ökologische Umstellung der Lebensführung nur wenig befördert. Gleichwohl bleibt die Frage offen, inwiefern nun das Konsumverhalten den Kriterien der Nachhaltigkeit entspricht und von welchen Determinanten es abhängt. Wir haben dazu die in der Studie „Zukunftsfähiges Deutschland“ (4) formulierten Kriterien für einen ressourcensparenden Konsum herangezogen:

- Sparsamkeit als ein überlegter, reflektierter Konsum;

- Langlebigkeit als eine Präferenz für qualitativ hochwertige, reparatur- und wartungsfreundliche Produkte mit einer langen Lebensdauer;

- Regionalorientierung sowohl in der Freizeitgestaltung als auch beim Einkauf und

gemeinsame Nutzung, also die Erhöhung der Gebrauchsintensität von Gütern.

Es hat sich gezeigt, dass diese Kriterien in jeweils sehr unterschiedlichem Maße in das Konsumverhalten Eingang gefunden haben. Entsprechende Verhaltensorientierungen wurden in den seltensten Fällen durch ökologische Reflexionen begründet, sondern in viel stärkerem Maße durch einen Rückbezug auf traditionelle Orientierungen in der Arbeiterschaft. 
Sparsamkeit hat zum Beispiel als eine Grundregel haushälterischen Handelns Niederschlag in der Lebensführung gerade von älteren Arbeitnehmerinnen und Arbeitnehmern gefunden. Es wurde häufig genau geprüft, ob eine größere Anschaffung notwendig war und auch bei alltäglichen Einkäufen wurde auf preisgünstige Produkte geachtet. Diese Sparsamkeit gründete sich nicht auf ethische Überlegungen, sondern auf Vorstellungen, die Zukunftsvorsorge einem kurzfristigen Konsum vorziehen oder auf eine gewisse Genügsamkeit abstellen. In Einzelfällen wurden reale Sparzwänge auch durch den Einkommensverlust verstärkt. Ebenfalls ließ sich ein positiver Bezug auf den Stellenwert von Produktqualität und Langlebigkeit finden, der sich gegen Ex-und-Hopp-Mentalitäten richtete.

Eine Regionalorientierung beim Einkauf und in der Freizeit hatte sich allerdings nur schwach niedergeschlagen. Kaum anschlussfähig an die Lebensfuihrung der befragten Industriebeschäftigten war auch die Idee einer gemeinsamen Nutzung von Gebrauchsgegenständen. Insgesamt ließen sich also die Muster eines nachhaltigen Konsums nur schwach und segmentiert in der alltäglichen Lebensführung wiederfinden. Bei Sparsamkeit und Langlebigkeit haben sich ökologisch bedeutsame, aber nicht intendierte Folgen aus Verhaltensmustern ergeben, die eingebunden sind in soziokulturelle Milieus und ihre teilweise traditionellen, aus früheren Knappheitserfahrungen überlieferten Normengefüge.

\section{Zwei Konsequenzen}

Im Resultat folgen daraus mindestens zwei wichtige Konsequenzen für die Ökologisierung von Konsumformen: Zum einen werden die Arrangements der alltäglichen Lebensfiuhrung stark durch Systemvorgaben und Gelegenheitsstrukturen geprägt, die auf die Möglichkeiten und Grenzen für die Etablierung von nachhaltigen Konsummustern einwirken. Die Dynamiken des Erwerbssektors und deren Anforderungen an den Einzelnen haben dabei einen zentralen Stellenwert, entsprechend sind diese Schnittstellen im Hinblick auf Gestaltungskonzepte sehr viel stärker in den Blick $\mathrm{zu}$ nehmen. Das gilt vor allem für die weitere Gestaltung und Absicherung der Erwerbsarbeit. Ein weiterer Ansatzpunkt könnten die neuen Versuche sein, Zeitpolitik als ein neues gesellschaftliches Politikfeld zu etablieren (5).

Zum zweiten stehen die Appelle an einen ressourcensparenden Konsum und die traditionellen Sparsamkeitstugenden bestimmter Milieus bisher unverbunden nebeneinander. Das heißt, bestimmte Suffizienzleistungen werden einerseits nicht anerkannt, während andererseits die ökologische Argumentation der Leitbilder bei manchen Bevölkerungsschichten kaum greift. Hier wäre zu prüfen, inwieweit die Vorschläge für ein ökologisches Konsumverhalten entlang der in den verschiedenen sozialen Milieus unterschiedlichen Praktiken der Lebensführung differenziert und zugespitzt werden können. Dazu gehört auch die Reflexion der Frage, unter welchen sozialen Voraussetzungen überhaupt Suffizienzleistungen möglich sind, welche sozialen Gruppen bereits weitgehende
Verzichtsleistungen erbringen und bei welchen Gruppen weitergehende Suffizienzstrategien ansetzen können.

\section{Anmerkungen}

(1) Das Forschungsprojekt „Arbeitsstile - Lebensstile Nachhaltigkeit” wurde mit Unterstützung der Hans-BöcklerStiftung von 1996 bis 1998 durchgeführt. Vgl. Hielscher, Volker/ Hildebrandt, Eckart: Zeit für Lebensqualität. Auswirkungen verkürzter und flexibilisierter Arbeitszeiten auf die Lebensführung. Berlin 1999.

(2) Der dem Projekt zugrundeliegende Ansatz der „reflexiven Lebensführung" knüpft an das Konzept der "alltäglichen Lebensführung" an. Vgl. Hildebrandt, Eckart (Hg.): Reflexive Lebensführung. Berlin, im Erscheinen; Voss, Gerd G.: Lebensführung als Arbeit. Über die Autonomie der Person im Alltag der Gesellschaft. Stuttgart 1991 sowie Projektgruppe „Alltägliche Lebensführung” (Hg.): Alltägliche Lebensführung. Arrangements zwischen Traditionalität und Modernisierung. Opladen 1995.

(3) In Anlehnung an die Wortschöpfung von VW-Personalvorstand Peter Hartz. Vgl. Hartz, Peter: Das atmende Unternehmen. Jeder Arbeitsplatz hat einen Kunden.

Frankfurt a.M./ New York 1996.

(4) Vgl. BUND/ Misereor (Hrsg.): Zukunftsfähiges Deutschland. Ein Beitrag zu einer global nachhaltigen Entwicklung. Basel 1996, S. 206-224.

(5) Vgl. Eberling, Matthias/ Henckel, Dietrich: Kommunale Zeitpolitik. Veränderung von Zeitstrukturen - Handlungsoptionen für Kommunen. Berlin 1998.

\section{Der Autor}

Volker Hielscher arbeitet als Sozialwissenschaftler am Wissenschaftszentrum Berlin für Sozialforschung (WZB) (Forschungsschwerpunkt Technik-Arbeit-Umwelt).

Kontakt: WZB, Reichpietschufer 50, 10785 Berlin, Tel. 030/25491-270, E-mail: hielscher@wz-berlin.de

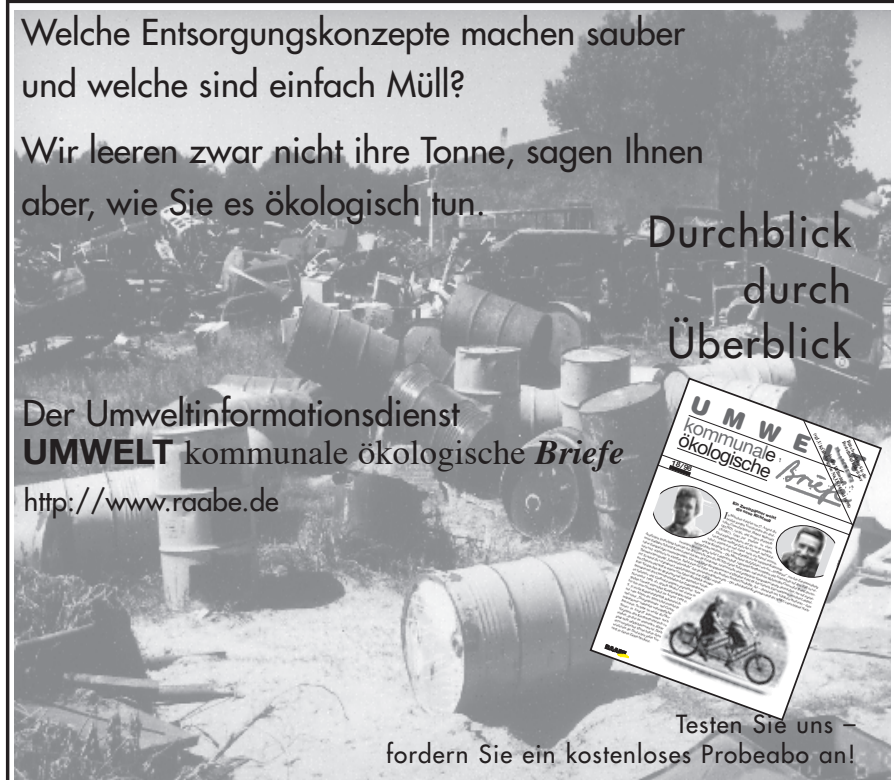


(c) 20I0 Authors; licensee IÖW and oekom verlag. This is an article distributed under the terms of the Creative Commons Attribution Non-Commercial No Derivates License (http://creativecommons.org/licenses/by-nc-nd/3.o/), which permits unrestricted use, distribution, and reproduction in any medium, provided the original work is properly cited. 